\title{
The importance of a 3D-based approach with personalized models for accurately assessing total body surface area
}

\author{
Adrien Desbois , MS, ${ }^{*} \uparrow$ Svetlana Matei , MD, + Isabelle Perreault , MD, $†$ \\ Florian Beguet, MS, ${ }^{*}+$ Sylvie Gervais , PhD, ${ }^{*}$ Jacques A de Guise , PhD, ${ }^{* \dagger}$ \\ *École de technologie supérieure (ÉTS), Département de genie de la production automatisée, Montréal, Québec, Canada; \\ * Laboratoire de recherche en imagerie et orthopédie (LIO), Centre de Recherche du CHUM, Montréal, Québec, Canada; \\ $\mp$ University of Montreal, Faculty of Medecine, Department of Surgery, Division of Plastic Surgery, Montreal, Quebec,
} Canada

\begin{abstract}
Objective:

In this paper, the relevance of a new three-dimensional computer-based framework with personalized 3D models for accurately assessing the TBSA is demonstrated through preliminary results and validation studies.

\section{Methods:}

First, a 3D rendering interface was developed for representation and calculation of TBSA. The personalized 3D models were built from anthropometric measurements using MakeHuman software. 15 paired models were randomly built with an equal body surface area but a different morphology. The difference of local body surface area, TBSA burned and Parkland fluids between each paired models were observed to highlight the impact of morphology's variation on the TBSA. Finally, a preliminary validation study was made on 4 mannequins by 14 volunteers to assess the accuracy of the 3D models built with MakeHuman software and TBSA burned assessment with the proposed method.

\section{Results:}

Small variations in the morphology impacted the TBSA assessment. Mannequin's 3D models built with MakeHuman software presented an absolute error of $3 \pm 2.2 \%$ with no significant difference with their scans. The proposed approach allows for a better assessment of TBSA with a lower variability. No significant difference in the scores for expert and non-expert conditions was observed.

\section{Conclusion:}

Personalized 3D model to the patient's morphology is suggested to overcome the difficulty of patients with specific morphologies such as obese and children. The proposed framework appears to be relevant for personalizing and accurately assessing TBSA and could reduce morbidity and mortality.
\end{abstract}

Keywords: TBSA, body surface area, burned body surface area, 3D model, anthropometry, anthropometric measures, morphology, validation.

*Corresponding author.

Jacques A de Guise, PhD, Laboratoire de recherche en imagerie et orthopédie (LIO), Centre de Recherche duCHUM, Montréal, Québec, Canada. Canada.

Email: jdeguise@etsmtl.ca. 


\section{Introduction}

Percent TBSA burned assessment is crucial for patients with major burn injuries. It helps not only in determining the quantity of fluid required but also in identifying criteria for transfer to a burn unit and evaluating the patient's outcome.

Many studies have highlighted the imprecision of the two-dimensional (2D) clinical methods for TBSA assessment due to the fact they require transposing three-dimensional (3D) burns onto a 2D diagram. According to some authors, clinical methods overestimate TBSA by $50 \%$ to $161 \%$ due to morphological variations of the patients, with a variability among observers of more than $62 \%{ }^{1-3}$. This is even more critical in the case of patients with central obesity ${ }^{4}$. However, obesity is a frequent clinical condition ${ }^{5}$ and is a common problem for all developed countries, where obesity rates are continuously increasing. Although children with burns represent the cases with the highest mortality, the outcomes for obese children are even worse ${ }^{6}$. Also, body proportions are not specific to the patient. The rule of nines is not appropriate for children ${ }^{6}$. The rule of palms can be ambiguous. For some authors, the surface of a palm includes the fingers ${ }^{7,8}$ while for others it does $\operatorname{not}^{9}$ or represents $0.78 \%$ of the body surface area (BSA) rather than $1 \%{ }^{10}$. The Lund \& Browder chart is the most appropriate method since it is adjusted for children ${ }^{2}$. Errors in assessing TBSA can lead to significant medical and surgical complications $^{2,6}$.

Given these challenges, many authors have attempted to improve TBSA assessment by developing digital methods. The first software applications were mainly digital representations of the Lund \& Browder chart, with automatic computation of TBSA and additional functions (fluid requirement, types of burns, etc.) such as SAGEII ${ }^{11}$ and BurnCalculator ${ }^{12}$. The latter has been shown to overestimate the burn area in $25 \%$ of cases and underestimate it in $15 \%$ of cases. Some efforts have been made to improve the accuracy of the Lund \& Browder chart by providing a more accurate hierarchical decomposition of the diagram ${ }^{13}$. As they still rely on a 2D diagram, these programs suffer from the same accuracy problems already mentioned above. To address this shortcoming, some authors have proposed representing burns in 3D. One of the first tools to achieve this aim was EPRI's 3D Burn Vision $^{11}$, which provides a single predefined 3D mesh. Although it affords a 3D representation of the burns, the mesh is not accurate and is difficult to adapt to the patient. The most known and reliable is BurnCase 3D ${ }^{3}$, 14-16 which uses a database of predefined 3D human models to supply an adapted 3D model based on the input of information such as age, gender, size, and weight. It computes TBSA and fluid requirement and has additional features (clinical records, images, etc.). However, a limitation of BurnCase 3D is the modeling algorithm which is based on empirical formulas to approximate the BSA of its adapted 3D models. These formulas can approximate BSA if weight and height are known ${ }^{10}, 17,18$. The TBSA assessment mainly depends on the BSA approximation which presents estimation error ${ }^{19}$. This is the case of the clinical methods which come from data compiled using these formulas ${ }^{10,20}$. A later solution, $\mathrm{BAI}^{21}$, improved the same process of selecting a model from a database of predefined 3D human models by including some obese 3D models. Thus far, BurnCase $3 \mathrm{D}^{3,14-16}$ and $\mathrm{BAI}^{21}$ have been the only tools to offer a suitable solution for assessing TBSA. However, the fact that predefined models are only adapted to the patient's weight and height means there is an unknown offset between the patient's real BSA and the BSA of the 3D model, which has not been evaluated ${ }^{22}$. The model selected is still an approximation of the patient's actual body constitution which sometime diverges from it's real $\mathrm{BSA}^{14}$. Nevertheless, the studies of Giretzlehner et $\mathrm{al}^{3}$ and Parvizi et $\mathrm{al}^{16}$ have highlighted the potential of computer-aider systems such as BurnCase 3D to improve TBSA assessment and inter-rater reliability for burns below $20 \%$ TBSA. A great improvement was the semiautomatic superimposition onto the 3D model of wound photography to help the tracing of burns. As well, the database offers a knowledge base of medical advice and diagnosis support. Several attempts have been made to capture the real shape of a person with a Kinect ${ }^{23}, \quad 24$ and thus provide personalized 3D models. Although the resulting 3D scan is personalized, it is not useable due to the Kinect's insufficient optical resolution, which necessitates post-treatment of the scan. The lengthy acquisition time $(>15 \mathrm{~min})$, the impossibility of moving the subject, and lack of interface make this method unsuitable at this time for initial management of burn patients in a clinical context.

An improvement to these tools would be to take into account the correlation between different human morphologies. As suggested by Dirnberger, Giretzlehner ${ }^{14}$, another approach whose model deformation algorithm relies on measurements entered by the surgeon should be considered.

In this paper we present a new 3D computer-based framework for evaluating TBSA, incorporating anthropometric measurements, MakeHuman (M.H.) software, and a 3D rendering interface. This approach aims to satisfy clinical requirements by maximizing TBSA assessment accuracy and personalizing 3D models of burn patients. The goals of this study were: first, to demonstrate the importance of personalizing 3D models to the patient's body constitution for a better assessment of TBSA. 


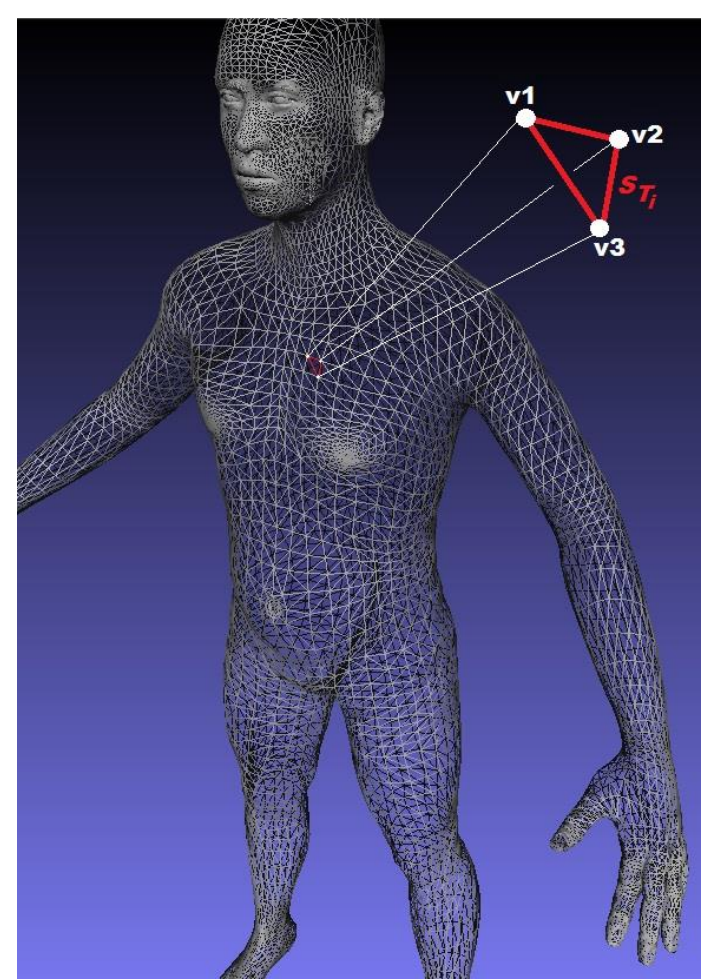

Figure 1. Example of the mesh model. $\mathrm{S}_{\mathrm{Ti}}$ is the surface area of 1 triangle and $\mathrm{v} 1, \mathrm{v} 2, \mathrm{v} 3$ its the adjacent vertices.

Second, to perform a preliminary validation of the 3D models built with the MakeHuman software. Third, to carry out a preliminary validation of the proposed approach.

\section{Methods}

\subsection{Burn representation and calculation of the $B S A, B B S A$ and TBSA}

To assess accurately the body surface area (BSA), burned body surface area (BBSA) and TBSA, a simple 3D rendering interface was developed in $\mathrm{C}++$ using Qt and VTK libraries.

The 3D model is composed of triangles (see Fig.1), each one composed of adjacent vertices v1, v2 and v3. The surface area $S_{T_{\mathrm{i}}}$, of a triangle $T_{\mathrm{i}}$ of the mesh, is calculated by Heron's formula ${ }^{[25]}$ based on the coordinates of the adjacent vertices (Eq. 2).

Using the computer mouse, the user can paint on the 3D model and therefore select the triangles which represent the BBSA. The BBSA is obtained using Eq. 1 for the painted triangles. The Non-BBSA is obtained using Eq. 1 for the non-painted triangles.

The BSA is obtained by the sum of the BBSA and non-BBSA. TBSA is obtained by dividing BBSA by the BSA.

$$
\begin{aligned}
& \mathrm{T}_{\mathrm{i}-\mathrm{v} 1}=\left(\mathrm{xT}_{\mathrm{i}-\mathrm{v} 1}, \mathrm{yT}_{\mathrm{i}-\mathrm{v} 1}, \mathrm{zT}_{\mathrm{i}-\mathrm{v} 1}\right), \\
& \mathrm{T}_{\mathrm{i}-\mathrm{v} 2}=\left(\mathrm{xT}_{\mathrm{i}-\mathrm{v} 2}, \mathrm{yT}_{\mathrm{i}-\mathrm{v} 2}, \mathrm{zT}_{\mathrm{i}-\mathrm{v} 2}\right) \\
& T_{\mathrm{i}-\mathrm{v} 3}=\left(\mathrm{xT}_{\mathrm{i}-\mathrm{v} 3}, \mathrm{yT}_{\mathrm{i}-\mathrm{v} 3}, \mathrm{zT}_{\mathrm{i}-\mathrm{v} 3}\right)
\end{aligned}
$$

represent the three coordinate sets of the adjacent vertices of the triangle $T_{i}$.

$\mathrm{A}_{\mathrm{T}_{\mathrm{i}-\mathrm{v} 1 \mathrm{v} 2}}, \mathrm{~B}_{\mathrm{T}_{\mathrm{i}-\mathrm{v} 1 \mathrm{v} 3}}$ and $\mathrm{C}_{\mathrm{T}_{\mathrm{i}-\mathrm{v} 2 \mathrm{v} 3}}$ represent the lengths of each side of the triangle $\mathrm{T}_{\mathrm{i}}$.

$\mathrm{P}_{\mathrm{T}_{\mathrm{i}}}=\frac{\left(\mathrm{A}_{T_{\mathrm{i}-\mathrm{v} 1 \mathrm{v} 2}}+\mathrm{B}_{T_{\mathrm{i}-\mathrm{v} 1 \mathrm{v} 3}}+\mathrm{C}_{T_{\mathrm{i}-\mathrm{v} 2 \mathrm{v} 3}}\right)}{2}$ represents the semi perimeter of triangle $\mathrm{T}_{\mathrm{i}}$.

$$
S=\sum_{1}^{n} S_{T_{i}}
$$

$\mathrm{S}_{T_{\mathrm{i}}}=\sqrt{\mathrm{P}_{T_{\mathrm{i}}}\left(\mathrm{P}_{\mathrm{T}_{\mathrm{i}}}-\mathrm{A}_{T_{\mathrm{i}-\mathrm{v} 1 \mathrm{v} 2}}\right)\left(\mathrm{P}_{\mathrm{T}_{\mathrm{i}}}-\mathrm{B}_{T_{\mathrm{i}-\mathrm{v} 1 \mathrm{v} 3}}\right)\left(\mathrm{P}_{\mathrm{T}_{\mathrm{i}}}-\mathrm{C}_{T_{\mathrm{i}-\mathrm{v} 2 \mathrm{v} 3}}\right)}$

\subsection{Importance of personalizing $3 D$ models}

To assess TBSA accurately with a 3D personalized model of a burn patient, the cross-platform opensource software MakeHuman ${ }^{\mathrm{TM}}$ (M.H.) ${ }^{26}$ was used. Based on a technical and artistic study of morphological features of the human body, this software includes a system for simulating disproportion of fat and muscle, allowing realistic modeling of a whole range of human anatomical shapes (see Fig.2).

The initial androgynous 3D model can be deformed into a realistic human model based on simple and intuitive parameters such as age, sex, height, and anthropometric measures. One of the additional features of MakeHuman software allows to randomly vary these parameters, thereby generating random $3 \mathrm{D}$ models.

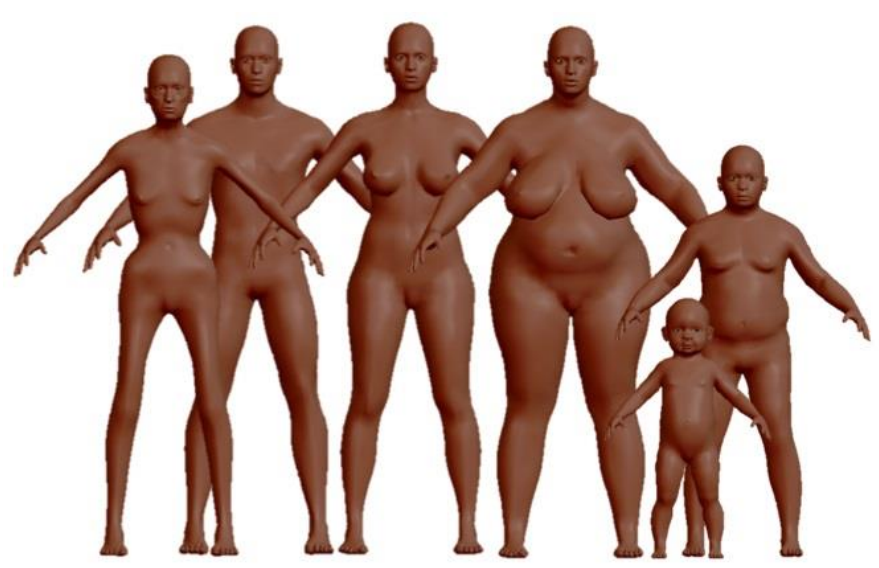

Figure 2. Examples of some three-dimensional models created with MakeHuman ${ }^{\mathrm{TM}}$ software. 

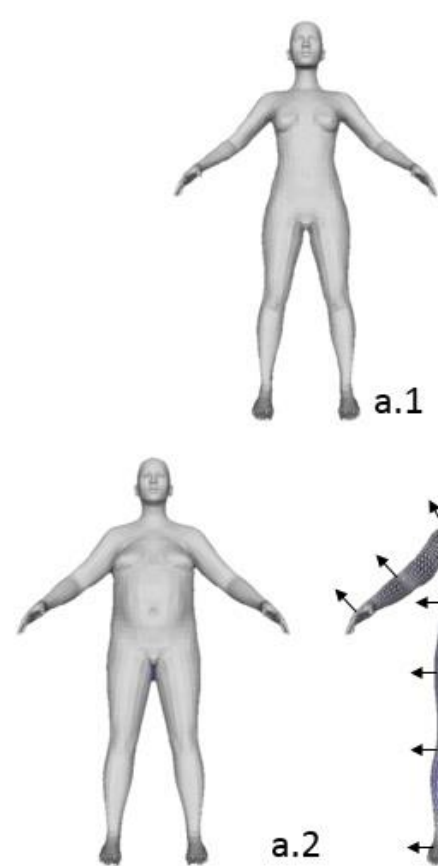
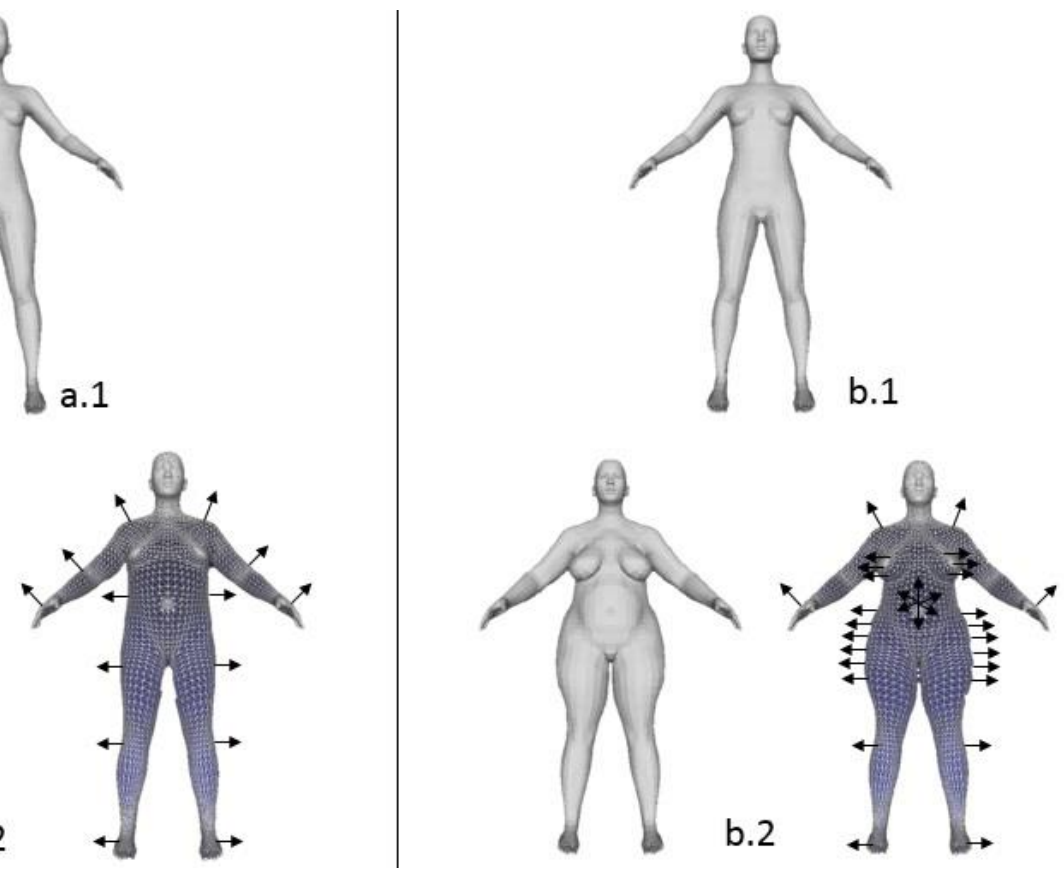

Figure 3: Difference between existing methods for 3D assessment of the TBSA and the proposed method. a.1 and b.1, Example of the base model. a.2, Global expansion algorithm of the model proposed by BurnCase $3 \mathrm{D}^{27}$ and $\mathrm{BAI}{ }^{21}$. b.2, Local expansion algorithm of MakeHuman software ${ }^{26}$ suggested in the proposed method. The represented models were built with the MakeHuman software and serve only as an example.

This study assumed that the typical features of the human morphology (e.g. android and gynecoid obesity, large breasts, fat disproportionality, etc.) can improve the assessment of TBSA. Indeed, as it can be seen in the Fig. 3, the modeling algorithm of BurnCase $3 \mathrm{D}^{14}$ and $\mathrm{BAI}^{21}$ are based on anthropometric formulas such as Eq. 3 below.

$$
\begin{gathered}
B S A=C \times W^{A} \times H^{B} A, B \text { and } C \text { are constants } \\
\text { defined by authors; } W=\text { weight; } H=\text { Height }
\end{gathered}
$$

The predefined 3D base model is first uniformly stretched along the longitudinal axis to fit the body height. Then, the model is adapted by expanding the surface along the normal vectors of the mesh polygons until the approximated BSA is reached. Because the assessment of TBSA mainly depends on the BSA approximated with the Eq. 3, the problem is that two human bodies with different morphology can produce two similar 3D projections (same BSA). To overcome this issue, the proposed approach suggests using M.H. software which locally expands its base model surface for each body parts depending on anthropometric measurements (see Fig. 3). The TBSA assessment depends therefore on the BSA but also the morphology which allows for more accuracy.

To verify this hypothesis, 15 paired models were first randomly generated. Paired models include two models with the same BSA but a different morphology (Fig. 4), which allows isolating the morphology's influence from the BSA. The random modeling algorithm of paired models follows these conditions: a first model is randomly generated. Its BSA is calculated with the Eq. 1 mentioned above. Then, a second model is randomly generated. Its calculated BSA must be equal to the BSA of the first model with an error margin of $\pm 0,01 \mathrm{~m}^{2}$. As well, its morphology must be different from the morphology of the first model. In total, 30 models (15 paired models), 19 men and 11 women models were randomly generated (see Table 1).

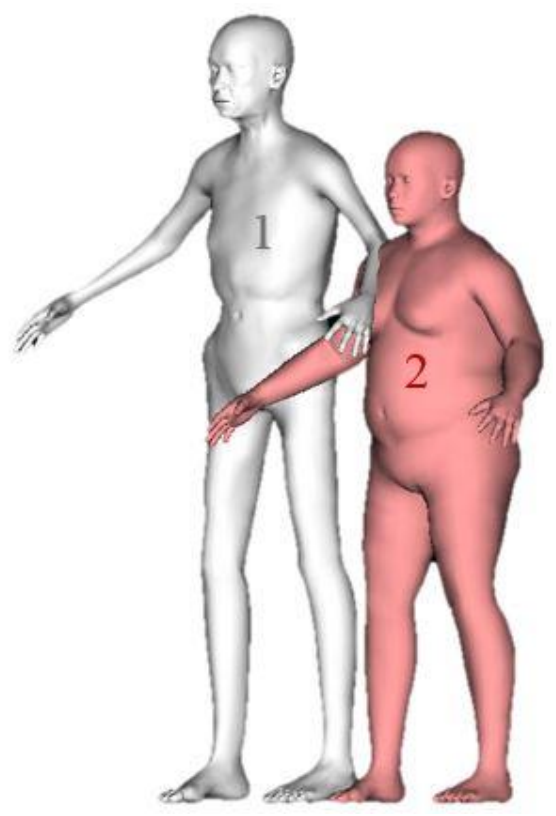

Figure 4: Example of paired models. The two models have the same BSA but a different morphology. 
Table 1: Summary of the paired models.

\begin{tabular}{lc}
\hline Characteristic & Value \\
Age (years) & $47 \pm 29$ \\
Height $(\mathrm{cm})$ & $166,73 \pm 18,14$ \\
Weight* $(\mathrm{kg})$ & $62 \pm 19$ \\
TBSA $\left(\mathrm{m}^{2}\right)$ & $1,7 \pm 0,3$ \\
\hline
\end{tabular}

Data presented as means \pm SD.

*Weight is the approximated weight with Eq 3 and 4.

Then, to quantify the impact of the body morphology on the TBSA and Parkland fluids, six consistent burn patterns were drawn manually using the $3 \mathrm{D}$ rendering interface mention above. The six burn patterns were drawn by a plastic surgeon and covered TBSA from $22 \%$ to $60 \%$ (see Fig. 5).

The six burn patterns (Fig. 5) were applied automatically and without control on the whole set of the $303 \mathrm{D}$ models.

Finally, for 2 paired models, the difference between both of BBSA and TBSA were estimated. Additionally, the differences in Parkland fluid requirements (Eq. 5) were estimated. This was estimated for the whole 15 paired models.

$$
* W=\left(\frac{B S A}{C \times H^{B}}\right)^{1 / A}
$$

$$
\begin{gathered}
\text { Fluids requirements }= \\
4 \mathrm{ml} \times \mathrm{TBSA}(\%) \times \text { Weigh }(\mathrm{kg})
\end{gathered}
$$

Because of the impossibility to quantify virtually the weight (which depends on the mass of the water, bones, muscle, fat, and so on) of the paired of models, the weight was approximate using Eq. 3 and 4 based on the study of Tikuisis et $\mathrm{al}^{19}$. This provides an overview of the impact of the variation of morphology on the fluids requirements.

The mean and standard deviation of the differences of BBSA, TBSA and Parkland fluids were automatically calculated with the $3 \mathrm{D}$ rendering interface regardless intra-inter variability and transposition error.

\subsection{Preliminary validation of the accuracy of the $3 D$ models built with MakeHuman software}

A preliminary validation study was made to assess the accuracy of the models built from anthropometric measures. First, 19 anthropometric measurements were manually taken on 4 commercial mannequins (Fig. 6) following a protocol. The protocol was based on ISO 7250-128 and measurer's handbook 29,30 .
The 19 measures taken have been logged in M.H. software to generate their respective 3D models. The accepted standard (which represents the true value) has been established by scanning the commercial mannequins (Fig. 6) with the white light portable 3D scanner Go!Scan ${ }^{\mathrm{TM}}$ of CREAFORM, Lévis, Québec, Canada. This scanner allows a resolution of $100 \mu \mathrm{m}$. The BSA of the five 3D scans and the five 3D models was obtained by the $3 \mathrm{D}$ rendering interface mentioned above.

The error percentage (Eq. 6) was estimated for each mannequin.

$$
\begin{gathered}
\% \text { Error }= \\
\left|\frac{B S A \text { model }- \text { BSA scan }}{B S A \text { scan }} \times 100\right|
\end{gathered}
$$

Using Statgraphics Centurion XVI software, a paired-samples $t$-test was performed to compare the mean BSA of the 3D scans to the mean BSA of the 3D models built with M.H software.

\subsection{Preliminary validation of the proposed approach}

Another preliminary validation study was made to assess the accuracy of the TBSA assessment made with the 3D rendering interface against the clinical method, the Lund \& Browder chart. Each method was compared with the gold standard of the TBSA and Parkland fluids of each mannequin.

First, patches with a known surface area $\left(\mathrm{m}^{2}\right)$ were put on the same commercial mannequin to simulate burns (Fig 6 left). Using the known BSA $\left(\mathrm{m}^{2}\right)$ of the mannequin's 3D scans (Fig. 6 right), the gold standard of the TBSA (\%) was estimated for each mannequin. Gold standards of Parkland fluids $(\mathrm{mL})$ were also estimated using Eq. 5 with the known approximated weight of the mannequin using Eq. 4 . Then, using the same 3D models of the mannequins previously built with M.H. software, 14 volunteers (8 experts and 6 non-experts) estimated the TBSA first using Lund \& Browder chart and then drawing on the 3D M.H. models with the 3D rendering interface. A modified Lund \& Browder chart was used for the child mannequin. A training to assess the TBSA on a Lund \& Browder chart was given to the non-experts. The 3D rendering interface automatically calculated the BSA, BBSA and TBSA in real time. This information was hidden to the volunteers. 


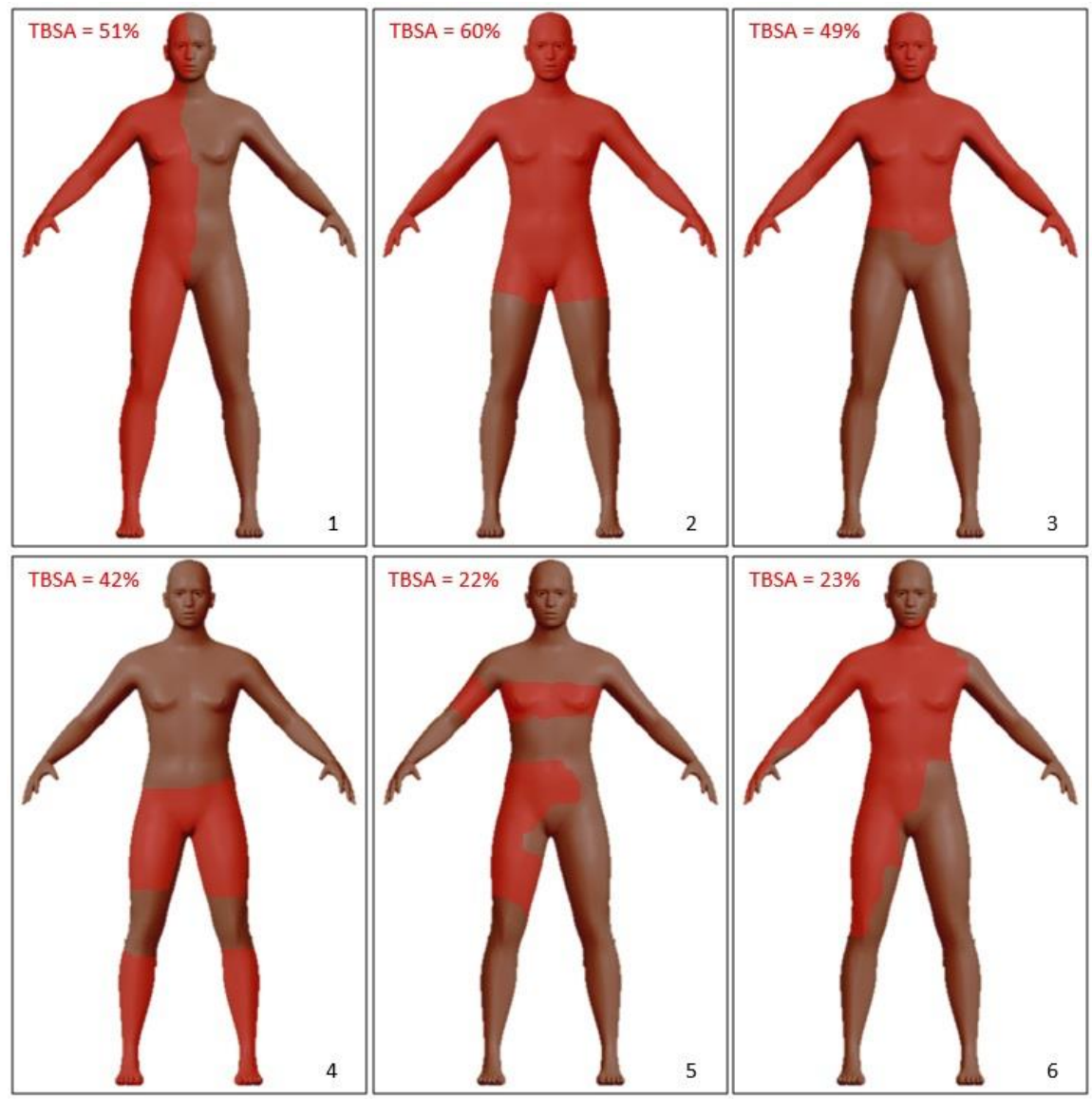

Figure 5: burn patterns used in this study. In red the Burned Body Surface Area (BBSA).

The error percentage for the TBSA assessment was calculated using Eq. 7 to measure the accuracy of each method.

$$
\begin{gathered}
\% \text { error TBSA }= \\
\left|\frac{\text { True TBSA }- \text { TBSA estimated }}{\text { True TBSA }} \times 100\right|
\end{gathered}
$$

Paired-samples t-test has been made with Statgraphics Centurion XVI software to assess if there was a significant difference for TBSA assessment between both methods.

The gain was calculated using Eq. 8 to measure the error reduction in the TBSA assessment when using the proposed approach over the clinical method.

$$
\begin{gathered}
\% \text { Gain TBSA }= \\
\left|\frac{\text { TBSA method }- \text { TBSA clinic }}{\text { True clinic }} \times 100\right|
\end{gathered}
$$

Finally, paired-samples t-test has been made to compare TBSA assessment made with the 3D rendering interface in experts and non-experts conditions.

\section{Results}

\subsection{Burn representation and calculation of the TBSA}

The 3D interface (Fig. 7) allows the user to upload a 3D model to standard 3D formats, draw the burns interactively using the mouse and obtain the resulting $\mathrm{BSA}$, non-BBSA and BBSA in square meter $\left(\mathrm{m}^{2}\right)$ and the TBSA in percentage $(\%)$. The BBSA is represented in red. The Non-BBSA in blue. The BSA is obtained by the sum of the BBSA and non-BBSA. TBSA is obtained by dividing BBSA by the BSA.

The user has access to various intuitive tools, such as paintbrush to paint or to un-paint the burns; translation, rotation, and scaling of the model along the three axes; and representation of the model as a surface or a mesh for better visibility. The latest release is stable on Windows and Macintosh.

\subsection{Importance of personalized $3 D$ models}

For all the burn patterns, the mean and standard deviation of the differences between each paired models were rather small, which was not the case of the TBSA. Only the burn pattern 1 showed small 


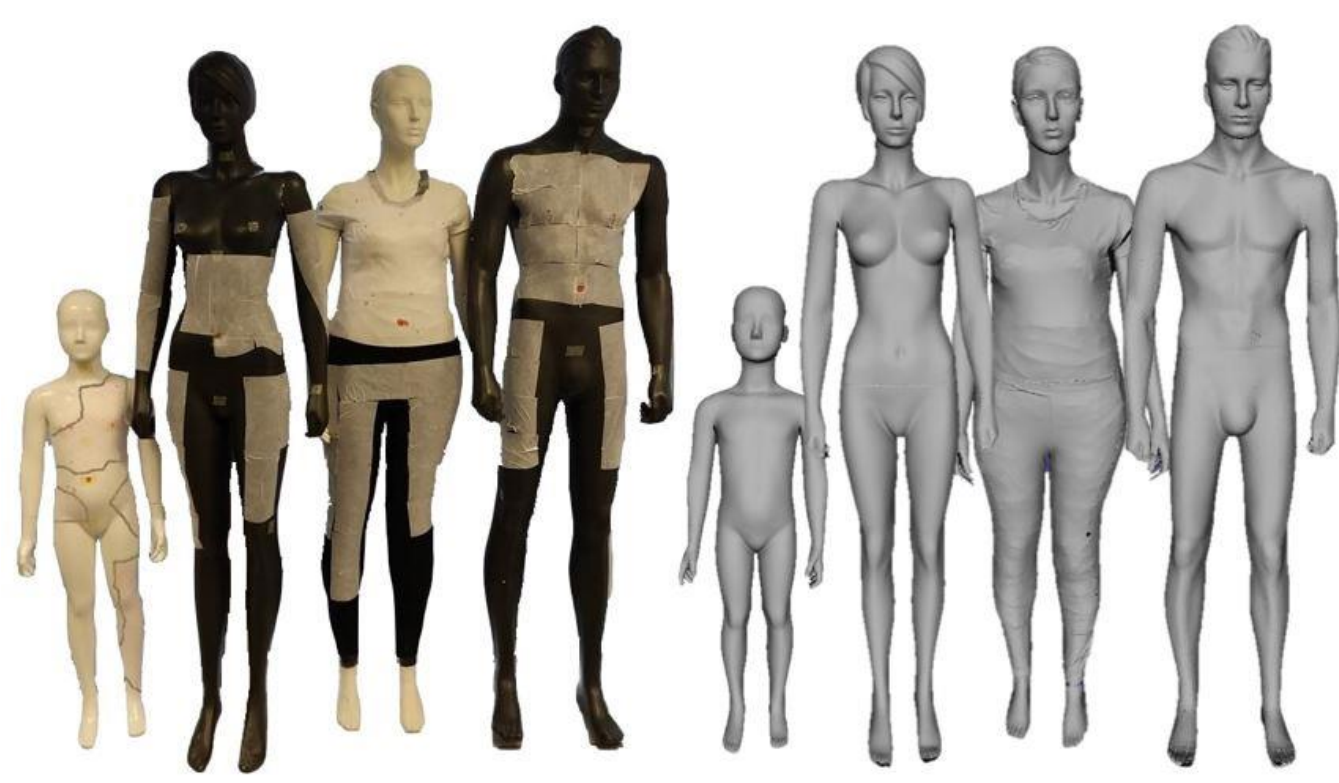

Figure 6: On the left, the 4 commercial mannequins used with patches represented simulated burns. On the right their respective 3D scans

variation. For all burn patterns, noticeable differences were observed.

For each paired models with the same BSA but a different morphology, small local variation in the morphology (characterized by the BBSA) influenced the TBSA and by consequence, the estimated Parkland fluids. The results are listed in the Table 2.
3.3. Preliminary validation of the accuracy of the $3 D$ models built with MakeHuman software

Results (see Table 3) have shown an average error percentage of $3 \pm 2.2 \%$ for the $\mathrm{BSA}$ of the MakeHuman models $(\mathrm{M}=1.53, \mathrm{SD}=0.45)$ in comparison with the BSA of the scans $(\mathrm{M}=1.51, \mathrm{SD}=0.48)$. A paired-samples t-test was conducted to compare the BSA in scans and MakeHuman models. There was not a significant difference between scans and MakeHuman models $(\mathrm{p}=0,4)$.

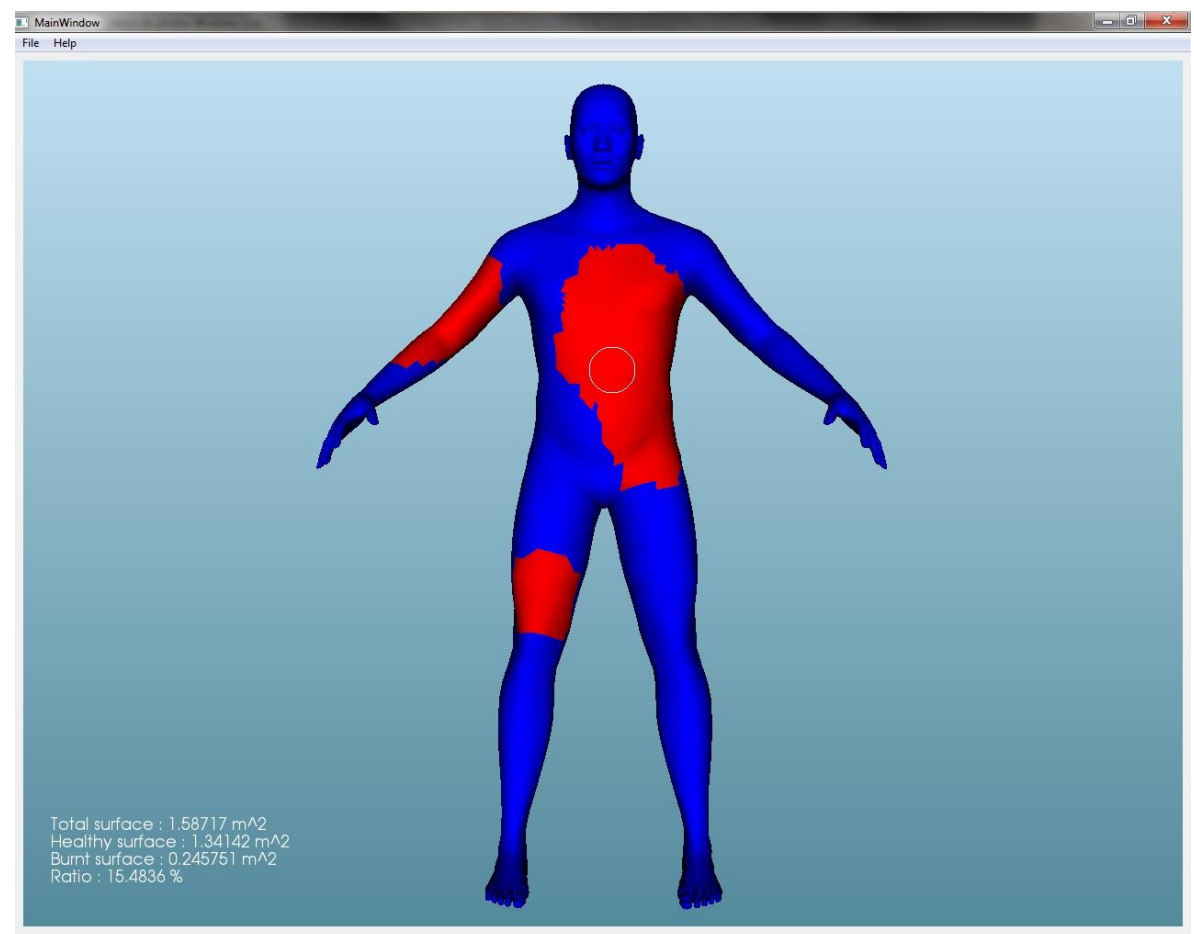

Figure 7: Example of the 3D rendering interface. 
Table 2: Mean and standard deviation of the difference of BBSA, TBSA and Parkland fluids between each paired models.

\begin{tabular}{cccc}
\hline Burn patterns & BBSA $\left(\mathrm{m}^{2}\right)$ & TBSA $(\%)$ & Fluids $(\mathrm{mL})$ \\
\hline 1 & $0,00 \pm 0,01$ & $0,27 \pm 0,2$ & $1271 \pm 1190$ \\
2 & $0,04 \pm 0,03$ & $1,98 \pm 1,40$ & $1746 \pm 1485$ \\
3 & $0,05 \pm 0,04$ & $2,73 \pm 2,15$ & $1537 \pm 1162$ \\
4 & $0,05 \pm 0,04$ & $2,99 \pm 2,14$ & $1257 \pm 1009$ \\
5 & $0.05 \pm 0.03$ & $2.58 \pm 1.76$ & $834 \pm 740$ \\
6 & $0.02 \pm 0.01$ & $1.20 \pm 0.72$ & $618 \pm 659$ \\
\hline \multicolumn{4}{c}{ Data presented as mean \pm SD. }
\end{tabular}

\subsection{Preliminary validation of the proposed approach}

The gold standards (true values) of the TBSA (\%) and estimated fluids for each mannequin are listed below in Table 4 .

On average, TBSA and Parkland fluids estimation (see Table 5) with the Lund \& Browder chart presented a greater variability among observers with a higher error percentage. Whereas TBSA and Parkland fluids estimation by drawing on the $3 \mathrm{D}$ models with the $3 \mathrm{D}$ rendering interface showed a lower variability and a lower absolute error (see Table 6).

Paired-samples t-test showed a significant difference between TBSA assessment with Lund \& Browder chart and with the 3D rendering interface for each mannequin $(\mathrm{p}<0,05)$. On average, the proposed approach allows a reduction error in TBSA assessment of $86 \%$ (see Table 6).

Paired-samples $t$-test was conducted to compare the assessment of the TBSA with the 3D rendering interface in experts and non-experts for each mannequin. There was not a significant difference in the scores for expert and nonexpert conditions for $\mathrm{P}>0,05$.

Table 3: summary of the BSA and error percentage of the scans and M.H. models

\begin{tabular}{lccccc}
\hline Mannequins & $\begin{array}{l}\text { Size } \\
(\mathrm{cm})\end{array}$ & $\begin{array}{l}\text { Weight } \\
(\mathrm{kg}) *\end{array}$ & $\begin{array}{l}\text { BSA } \\
\text { Scanner } \\
\left(\mathrm{m}^{2}\right)\end{array}$ & $\begin{array}{l}\text { BSA M.H. } \\
\left(\mathrm{m}^{2}\right)\end{array}$ & $\begin{array}{l}\text { Error } \\
(\%)\end{array}$ \\
\hline Child & 117 & 19 & 0.82 & 0.87 & 6 \\
$\begin{array}{l}\text { Woman } \\
\text { Overweight }\end{array}$ & 175.8 & 50 & 1.59 & 1.60 & 1 \\
$\begin{array}{l}\text { Woman } \\
\text { Man }\end{array}$ & 172 & 61 & 1.72 & 1.75 & 2 \\
\hline \multicolumn{5}{c}{ *The Weight is the approximated weight with Eq 3 and 4. } \\
\hline
\end{tabular}

\section{Discussions}

The 3D rendering interface presented in this study showed that it is easily possible in a simple and userfriendly manner to accurately assess the TBSA (\%) onto a 3D model.
Table 4: Accepted standard for the surface areas of the patches and estimated fluids for each mannequin.

\begin{tabular}{ccccc}
\hline Mannequins & $\begin{array}{c}\text { BBSA } \\
\left(\mathrm{m}^{2}\right)\end{array}$ & $\begin{array}{c}\text { BSA } \\
\left(\mathrm{m}^{2}\right)\end{array}$ & $\begin{array}{c}\text { TBSA } \\
(\%)\end{array}$ & $\begin{array}{c}\text { Fluids } \\
(\mathrm{mL})\end{array}$ \\
\hline Child & 0.2 & 0.8 & 23 & 1773 \\
Woman & 0.4 & 1.6 & 23 & 4534 \\
$\begin{array}{c}\text { Overweight } \\
\text { woman } \\
\text { man }\end{array}$ & 0.6 & 1.7 & 33 & 7962 \\
& 0.5 & 1.9 & 26 & 7925
\end{tabular}

BBSA: Burned Body Surface Area. BSA: Body Surface Area. TBSA: Percent Total Body Surface Area assessment.

Results obtained in section 3.2, point out the importance of taking the difference in human morphologies into consideration and so, looking for a 3D personalization of the models in the accurate assessment of TBSA. As shown in table 2, for the same BSA, small variations in the local body surface area (due to different morphology) can induce an important clinical variation in the TBSA and Parkland fluids assessment. This is the actual improvement that misses from the current suitable 3D tools for patients with major burn injuries such as BurnCase $3 \mathrm{D}^{3,14-16}$ and $\mathrm{BAI}^{21}$. In most case, the $\mathrm{BSA}$ is overestimated with a tendency to increase the size of the body ${ }^{31}$. An underestimation of the BSA of the obese person ${ }^{32}$ is also observed up to $20 \%{ }^{33}$. This can underestimate or overestimate morphology and therefore leads to an inadequate fluids estimation. BAI's predefined obese 3D models ${ }^{21}$ are insufficient to cover the variability of human morphology, specifically when the fat is disproportionally accumulated among obese persons or women with larger sized breasts ${ }^{6,34}$. As a matter of fact, the anterior trunk proportions of a woman's body can be altered by her breasts' size ${ }^{34}$. The same conditions are observed with the disproportion of the head of the children relative to their body. Moreover, Wells, Treleaven $^{35}$ have demonstrated the significant association between body mass index and chest and waist in men and bust and hips in women. These issues had led to the aim of the proposed approach that is to personalized $3 \mathrm{D}$ models based on the anthropometric measurements of the patients. By doing this, it is possible to reach the typical features of the human body shape. This can make a difference not only in accurate morphology modeling but also the BSA and therefore the TBSA. It is, even more, striking with the study of Klein, Hayden ${ }^{36}$ which has highlighted the medical and surgical complication that occur when fluid requirements assessment are under or overestimated by $1,5 \mathrm{~L}$. The risks are the development of multi-organ failure, bloodstream infections, pneumonia, respiratory distress syndrome and death. 
Table 5: summary of the Percent Total Body Surface Area (TBSA) assessment with the Lund \& Browder chart.

\begin{tabular}{lcccc}
\hline \multicolumn{1}{c}{ Mannequin } & Experts & Non-Experts & Estimated Fluids (mL) & Assessment Error (\%) \\
\hline Child & $31 \pm 3$ & $29 \pm 5$ & $2272 \pm 297$ & $30 \pm 12$ \\
Woman & $34 \pm 11$ & $31 \pm 4$ & $6509 \pm 1674$ & $44 \pm 37$ \\
Overweight woman & $40 \pm 6$ & $41 \pm 6$ & $9865 \pm 1352$ & $24 \pm 17$ \\
man & $37 \pm 7$ & $34 \pm 4$ & $10853 \pm 1835$ & $37 \pm 23$ \\
\hline
\end{tabular}

Data presented as mean \pm SD or percentage $(\%)$.

Results in section 3.3 have shown that M.H. 3D models are sufficiently accurate. The greatest error percentage obtained is observed for the child mannequin (Table 3). This can be explained by the fact that body shapes of the commercial mannequins do not correspond to real human body shape. Indeed, M.H. software takes correlation between body part and disproportion of morphology (e.g. the disproportion of the child's head as mentioned above) into consideration. The values of M.H. anthropometric measures are directly correlated among themselves allowing realistic 3D models. The difference of body dimensions does not allow to exactly build the respective $3 \mathrm{D}$ models of the mannequins. This limitation will be overcome later by validating the M.H. models directly to real subject scans. Nevertheless, these preliminary results allow seeing the suitability of M.H. software for this study.

Results in section 3.4 show a net decreased of the error percentage and variability in the estimation of the TBSA for the proposed approach against the Lund \& Browder chart regardless of the level of expertise (experts or non-experts). This confirms the relevance and interest of a new 3D computer-based framework for evaluating TBSA, incorporating anthropometric measurements, MakeHuman (M.H.) software, and a 3D rendering interface. As stated by Kamolz, Wurzer ${ }^{37}$, there is a need for objective burn size estimation such as 3D computer-aided system. A computer-aided system will help to not only compensate TBSA estimation errors but also provide an objective tool for reducing variability ${ }^{3}, 16$. An approach that focuses on personalizing 3D model is highly recommended, especially when we know that many clinical scores for burn patients (Abbreviated Burn Severity Index, R-Baux, etc.) use also TBSA (\%) for evaluating the patient's chances of survival or death. A slight change in the results using those scores can influence the level of care and the nature of the treatment provided ${ }^{38-41}$. An assessment of TBSA (\%) personalized would allow a better division of severe burned patients that really need to be taken care of.

This study presents several limitations. First, the constraint of using a triangle representation of a 3D model made the burn surface areas being roughly represented. This can hinder the TBSA assessment. Second, both validation of the 3D models of M.H. and the whole proposed method were made using 19 anthropometrics measurements. In a clinical practice, using only 4 to 5 anthropometrics measurements is feasible. Moreover, only the assessment of the TBSA was studied. The whole process of taking anthropometric measures on the patient, entering them into a software for the modeling of the 3D model and finally assessing the TBSA with the rendering interface was not evaluated.

In order to overcome these limitations, further work is intended to be done. First, to identify a limited set of the most influent, feasible in clinical practice, repeatable and reproducible anthropometric measurements on the human morphology. Second, the integration of a new $3 \mathrm{D}$ rendering interface. The painting of the burn surface will be done on 3D textures instead of a triangle representation, whichmean the painting will exactly follow the user's hand without constraint. Third, to validate the clinical study of the whole process of the method.

\section{Conflict of interest:}

None.

Table 6: summary of the Percent Total Body Surface Area (TBSA) assessment with the three-dimensional (3D) rendering

\begin{tabular}{lccccc}
\hline Mannequin & Experts & Non-Experts & $\begin{array}{c}\text { Estimated } \\
\text { Fluids (mL) }\end{array}$ & Assessment Error (\%) & Gain (\%) \\
\hline Child & $23 \pm 2$ & $24 \pm 2$ & $1786 \pm 142$ & $6 \pm 5$ & $80 \pm 58$ \\
Woman & $22 \pm 1$ & $22 \pm 1$ & $4443 \pm 256$ & $4 \pm 4$ & $91 \pm 89$ \\
Overweight woman & $32 \pm 0$ & $32 \pm 3$ & $7829 \pm 462$ & $4 \pm 4$ & $83 \pm 76$ \\
man & $25 \pm 1$ & $25 \pm 1$ & $7637 \pm 354$ & $4 \pm 4$ & $89 \pm 83$ \\
\hline
\end{tabular}

Data presented as mean \pm SD or percentage $(\%)$. 


\section{Acknowledgments}

This study was performed, thanks to financial support from NSERC and the Canada Research Chair of Jacques de Guise. The research team would like to thank the individuals who generously shared their time and experience for the purposes of this project.

\section{References}

1. Wachtel, T.L., et al., The inter-rater reliability of estimating the size of burns from various burn area chart drawings. Burns, 2000. 26(2): p. 156-170.

2. Parvizi, D., et al., The potential impact of wrong TBSA estimations on fluid resuscitation in patients suffering from burns: Things to keep in mind. Burns, 2014. 40(2): p. 241-245.

3. Giretzlehner, M., et al., The determination of total burn surface area: How much difference? Burns, 2013. 39(6): p. 11071113.

4. Kamolz, L.-P., et al., Burn surface area calculation: what do we need in future. Burns: journal of the International Society for Burn Injuries, 2014. 40(1): p. 171.

5. Neaman, K.C., et al., A new method for estimation of involved BSAs for obese and normal-weight patients with burn injury. Journal of Burn Care \& Research, 2011. 32(3): p. 421-428.

6. Livingston, E.H. and S. Lee, Percentage of Burned Body Surface Area Determination in Obese and Nonobese Patients. Journal of Surgical Research, 2000. 91(2): p. 106-110.

7. Hettiaratchy, S. and R. Papini, $A B C$ of burns: Initial management of a major burn: I-overview. BMJ: British Medical Journal, 2004. 328(7455): p. 1555.

8. Durrant, C.A.T., A.R. Simpson, and G. Williams, Thermal injury - The first $24 \mathrm{~h}$. Current Anaesthesia \& Critical Care, 2008. 19(5): p. 256-263.

9. Malic, C., et al., Resuscitation burn carda useful tool for burn injury assessment. Burns, 2007. 33(2): p. 195-199.

10. Gehan, E.A. and S.L. George, Estimation of human body surface area from height and weight. Cancer chemotherapy reports. Part 1, 1970. 54(4): p. 225-235.

11. Neuwalder, J., et al., A review of computeraided body surface area determination: SAGE II and EPRI's 3D Burn Vision. Journal of Burn Care \& Research, 2002. 23(1): p. 55-59.

12. Berry, M.G., et al., Digitisation of the total burn surface area. Burns, 2006. 32(6): p. 684-688.
13. Richard, R., J.A. Jones, and P. Parshley, Hierarchical Decomposition of Burn Body Diagram Based on Cutaneous Functional Units and Its Utility. Journal of Burn Care \& Research, 2015. 36(1): p. 33-43.

14. Dirnberger, J., Giretzlehner M, Luckeneder T, Siegl D, Haller HL, Rodemund C. BurnCase 3D-Realistic Adaptation of 3Dimensional Human Body Models, in Medical Image Computing and ComputerAssisted Intervention-MICCAI 2004. Lecture Notes in Computer Science, vol 3217. Springer, Berlin, Heidelberg, p. 363370.

15. Dirnberger, J., et al., Modelling human burn injuries in a three-dimensional virtual environment. Studies in health technology and informatics, 2002. 94: p. 52-58.

16. Parvizi, D., et al., BurnCase 3D software validation study: Burn size measurement accuracy and inter-rater reliability. Burns, 2016. 42(2): p. 329-335.

17. Du Bois, D. and E. Du Bois, A formula to estimate the approximate surface area if height and weight be known. 1916. Nutrition (Burbank, Los Angeles County, Calif.), 1989. 5(5): p. 303.

18. Haycock, G.B., G.J. Schwartz, and D.H. Wisotsky, Geometric method for measuring body surface area: a heightweight formula validated in infants, children, and adults. The Journal of pediatrics, 1978. 93(1): p. 62-66.

19. Tikuisis, P., P. Meunier, and C. Jubenville, Human body surface area: measurement and prediction using three dimensional body scans. European journal of applied physiology, 2001. 85(3-4): p. 264-271.

20. Lund, C.C. and N.C. Browder, The estimation of areas of burns. Surg Gynecol Obstet, 1944. 79(352): p. 8.

21. Prieto, M.F., et al., A system for $3 D$ representation of burns and calculation of burnt skin area. Burns, 2011. 37(7): p. 1233-1240.

22. Dirnberger, J., et al., Comments on " $A$ system for $3 D$ representation of burns and calculation of burnt skin area”. Burns, 2012. 38(7): p. 1091-1093.

23. Sheng, W.-b., et al., BurnCalc assessment study of computer-aided individual threedimensional burn area calculation. Journal of translational medicine, 2014. 12(1): p. 112.

24. Popescu, C.R. and A. Lungu, Real-Time 3D Reconstruction Using a Kinect Sensor. Computer Science and Information Technology (CS \& IT), 2014. 2(2): p. 9599. 
25. Weisstein, E.W., Heron's Formula. MathWorld, 2015.

26. Bastioni, M., S. Re, and S. Misra. Ideas and methods for modeling 3D human figures: the principal algorithms used by MakeHuman and their implementation in a new approach to parametric modeling. in Proceedings of the 1st Bangalore Annual Compute Conference. 2008. ACM, Bangalore, India.

27. Giretzlehner, M., Dirnberger, J., Luckender, T., Haller, H. L., \& Rodemund, C., BurnCase 3D: A research product for effective and time-saving documentation of burn injuries. Annals of Burns and Fire Disasters, 2004. 17(2): p. 64-72.

28. ISO, I., 7250-1: Basic Human Body Measurements for Technological DesignPart 1: Body Measurement Definitions and Landmarks. Geneva, Switzerland: International Organization for Standardization, 2008.

29. Hotzman, J., Gordon, C. C., Bradtmiller, B. et al., Measurer's Handbook: US Army and Marine Corps Anthropometric Surveys, 2010-2011. 2011, DTIC Document.

30. Gordon, C. C., Blackwell, C. L., Bradtmiller, B. et al., 2010 Anthropometric Survey of US Marine Corps Personnel: Methods and Summary Statistics. 2013, DTIC Document.

31. Bailey, B. and G. Briars, Estimating the surface area of the human body. Statistics in medicine, 1996. 15(13): p. 1325-1332.

32. Lee, J.-Y., J.-W. Choi, and H. Kim, Determination of Body Surface Area and Formulas to Estimate Body Surface Area Using the Alginate Method. Journal of PHYSIOLOGICAL ANTHROPOLOGY, 2008. 27(2): p. 71-82.

33. Tucker, G.R. and J.K. Alexander, Estimation of body surface area of extremely obese human subjects. Journal of applied physiology, 1960. 15(5): p. 781784.

34. Hidvegi, N., et al., Estimation of breast burn size. Plastic and reconstructive surgery, 2004. 113(6): p. 1591-1597.

35. Wells, J.C., P. Treleaven, and T.J. Cole, BMI compared with 3-dimensional body shape: the UK National Sizing Survey. The American journal of clinical nutrition, 2007. 85(2): p. 419-425.

36. Klein, M.B., et al., The association between fluid administration and outcome following major burn: a multicenter study. Annals of surgery, 2007. 245(4): p. 622-628.

37. Kamolz, L.-P., et al., Burn surface area calculation instead of burn size estimation: our opinion. Burns, 2014. 40(8): p. 18131814.

38. Ryan, C.M., et al., Objective estimates of the probability of death from burn injuries. New England Journal of Medicine, 1998. 338(6): p. 362-366.

39. Tahir, S., et al., Prediction of Mortality After Major Burn: Physiological Versus Biochemical Measures. Wounds, 2009. 21(7): p. 177-182.

40. Kraft, R., et al., Burn size and survival probability in paediatric patients in modern burn care: a prospective observational cohort study. The Lancet, 2012. 379(9820): p. 1013-1021.

41. Finnerty, C.C., et al., Hypertrophic scarring: the greatest unmet challenge after burn injury. The Lancet, 2016. 388(10052): p. 1427-1436. 